\title{
Propagação de Sólitons em Fibras Óticas Dissipativas
}

\author{
Caroline Dall' Agnol, Paulo Laerte Natti \\ UEL - Departamento de Matemática \\ 86051-990, Londrina, PR \\ E-mail: carol_dall@hotmail.com, plnatti@uel.br \\ Eliandro Rodrigues Cirilo, Neyva Maria Lopes Romeiro \\ UEL - Departamento de Matemática \\ 86051-990, Londrina, PR \\ E-mail: ercirilo@uel.br, nromeiro@uel.br
}

\begin{abstract}
Resumo: Toda a propagação de onda apresenta algum tipo de perda e, por menor que seja esta perda, gerará o decaimento desta onda. Dessa forma, o principal objetivo deste trabalho é estudar a perda de potência de ondas do tipo sóliton durante a propagação através de fibras óticas dissipativas e avaliar o impacto desta dissipação na transmissão do sinal sóliton. Neste contexto, foi desenvolvido um procedimento numérico para resolver um sistema de equações diferenciais parciais que descrevem esta propagação. Este procedimento foi fundamentado no método das diferenças finitas e no método de Gauss-Seidel com relaxação e através dele é possível observar e analisar o decaimento da onda sóliton, enquanto esta se propaga.
\end{abstract}

Palavras-chave: Sóliton, Comunicação Ótica, Dissipação

Uma das atividades essenciais da condição humana é sem dúvida a comunicação. Comunicase para trocar e expor ideias, fazer contatos e relatar fatos. A troca de informações tornou-se de suma importância tanto para a vida pessoal quanto para a vida profissional. Quando se trata de fluxo de dados, esta troca de informações pode ocorrer de forma analógica ou digital através de diversos meios, tais como cabos coaxiais, microondas, rádio frequências, infravermelho e fibras óticas. Com o acesso à internet facilitado através de celulares, notebooks e tablets vemos crescer a cada dia o tráfego de informações o que obriga os sistemas de comunicação a terem maior capacidade de transmissão, largura de banda, eficiência e rapidez sem perdas significativas de energia e com baixos custos [1].

Dentre os meios citados de transmisssão de dados, os sistemas de comunicação por fibra ótica se mostram os mais adequados à estas exigências, pois apresentam baixo fator de atenuação, grande largura de banda, baixos custos de produção e de armazenamento, devido ao seu formato leve, flexível e pouco volumoso; elevada resistência elétrica o que as torna um meio altamente isolante, impedindo interferências eletromagnéticas externas e garantindo melhor qualidade de transmissão, com sigilo, entre outras vantagens [4].

Porém, as características físicas da fibra ótica são muito importantes para a preservação da qualidade do sinal transmitido, de modo que inomogeneidades, difusão de moléculas de hidrogênio, bolhas na fibra, variações de diâmetro e rugosidades podem perturbar os sinais, gerando ruídos e perda de energia. Por isso, nas últimas décadas foram realizadas várias experiências no sentido de compensar a dispersão e as não-linearidades em sistemas de comunicação ótica em grandes distâncias a fim de aumentar a sua capacidade de transmissão de dados. Uma das mais importantes inovações na área de tecnologia da comunicação, que pode ser capaz de contornar essas dificuldades, baseia-se no conceito de sóliton ótico [4].

Os sólitons são pulsos óticos cuja envolvente do campo elétrico tem a forma de secante 
hiperbólica com alguns miliwatts de potência de pico capazes de se propagarem mantendo inalterada a sua forma, num meio não-linear e dispersivo como a fibra ótica. O princípio dos sólitons em fibras óticas fundamenta-se no perfeito equilíbrio entre o a velocidade de dispersão de grupo (Group Velocity Dispersion - GVD) e o efeito Kerr [3].

Smith et al. (1996) mostraram que um sóliton pode se propagar numa fibra com variação periódica de dispersão, mesmo que a dispersão média fosse praticamente nula, desse modo surgiu uma nova ideia para sistemas com sólitons: os sistemas com dispersão gerida [5]. Outras experiências foram realizadas por Fukuchi et al (1999), em sistemas mono-canal e WDM, visando alcançar a capacidade de transmissão de 1 Tb/s [2]. Em 2000 a Algety Telecom, instalada em Lannion, na França, tornou o uso prático de sólitons realidade desenvolvendo equipamentos de telecomunicações submarinos baseados na transmissão de sólitons óticos.

Atualmente, muitas pesquisas têm sido realizadas no intuito de encontrar um sistema solitônico capaz de competir comercialmente com os sistemas atuais de comunicação, ou seja, que ofereçam altas taxas de transmissão a baixo custo. Nesse contexto foram realizados estudos numéricos que descrevem a propagação de sólitons em fibras óticas dielétricas, dando ênfase ao estudo da perda de potência e à avaliação do impacto dessa dissipação na transmissão do sinal sóliton [3].

As equações de Maxwell descrevem a propagação de feixes óticos em guias de onda. A partir dessas equações obtemos o sistema acoplado de EDP's não-lineares, complexas, que descrevem a evolução longitudinal de duas ondas eletromagnéticas acopladas em um guia dielétrico, com não-linearidades quadráticas, chamado de dielétrico do tipo $\chi^{(2)}$ :

$$
\begin{aligned}
i \frac{\partial a_{1}}{\partial \xi}-\frac{r}{2} \frac{\partial^{2} a_{1}}{\partial s^{2}}+a_{1}^{*} a_{2} \exp (-i \beta \xi) & =0 \\
i \frac{\partial a_{2}}{\partial \xi}-i \delta \frac{\partial a_{2}}{\partial s}-\frac{\alpha}{2} \frac{\partial^{2} a_{2}}{\partial s^{2}}+a_{1}^{2} \exp (i \beta \xi) & =0 .
\end{aligned}
$$

Nestas equações $i=\sqrt{-1}$ é a unidade imaginária, $a_{1}(\xi, s)$ e $a_{2}(\xi, s)$ são funções complexas que representam as amplitudes normalizadas dos campos elétricos das ondas fundamental e segundo harmônico, respectivamente, e $a_{1}^{*}(\xi, s)$ é o conjugado complexo. A variável independente $s$ tem caráter de dimensão espacial, enquanto que a variável independente $\xi$ tem caráter temporal. Os parâmetros reais $\alpha, \beta, \delta$ e $r$ estão relacionados com as propriedades dielétricas da fibra ótica.

O sistema de EDP's (1) possui soluções do tipo sóliton dadas por [3]

$$
\begin{aligned}
a_{1}(\xi, s)= & \pm \frac{3}{2(\alpha-2 r)} \sqrt{\alpha r}\left(\frac{\delta^{2}}{2 \alpha-r}+\beta\right) \\
& \times \operatorname{sech}^{2}\left[ \pm \sqrt{\frac{1}{2(2 r-\alpha)}\left(\frac{\delta^{2}}{2 \alpha-r}+\beta\right)}\left(s-\frac{r \delta}{2 \alpha-r} \xi\right)\right] \\
& \times \exp \left\{i\left[\frac{r \delta^{2}(4 r-5 \alpha)}{2(2 \alpha-r)^{2}(2 r-\alpha)}-\frac{r \beta}{2 r-\alpha}\right] \xi-\frac{i \delta}{2 \alpha-r} s\right\} \\
a_{2}(\xi, s)= \pm & \frac{3 r}{2(\alpha-2 r)}\left(\frac{\delta^{2}}{2 \alpha-r}+\beta\right) \\
& \times \operatorname{sech}\left[ \pm \sqrt{\frac{1}{2(2 r-\alpha)}\left(\frac{\delta^{2}}{2 \alpha-r}+\beta\right)}\left(s-\frac{r \delta}{2 \alpha-r} \xi\right)\right] \\
& \times \exp \left\{2 i\left[\frac{r \delta^{2}(4 r-5 \alpha)}{2(2 \alpha-r)^{2}(2 r-\alpha)}-\frac{r \beta}{2 r-\alpha}+\frac{\beta}{2}\right] \xi-\frac{2 i \delta}{2 \alpha-r} s\right\} .
\end{aligned}
$$

A existência destas soluções está condicionada ao fato do argumento da função secante hiperbólica ser real e à não-existência de singularidades em (2) e (3). 
Como sabemos, a propagação de ondas tipo sólitons em fibras óticas reais não ocorre da mesma forma que em fibras óticas ideais. Existem muitos processos que podem causar perturbações na propagação. Assim, a fim de observar a perda de energia, causada por esses processos na onda sóliton, acrescentamos dois termos perturbativos à equação (1):

$$
\begin{aligned}
i \frac{\partial a_{1}}{\partial \xi}-\frac{r}{2} \frac{\partial^{2} a_{1}}{\partial s^{2}}+a_{1}^{*} a_{2} \exp (-i \beta \xi) & =i \mu a_{1}+i \kappa\left|a_{1}\right|^{2} a_{1} \\
i \frac{\partial a_{2}}{\partial \xi}-i \delta \frac{\partial a_{2}}{\partial s}-\frac{\alpha}{2} \frac{\partial^{2} a_{2}}{\partial s^{2}}+a_{1}^{2} \exp (i \beta \xi) & =i \mu a_{2}+i \kappa\left|a_{2}\right|^{2} a_{2}
\end{aligned}
$$

onde os termos $i \mu a_{1}$ e $i \mu a_{2}$ são termos dissipativos lineares e $i \kappa\left|a_{1}\right|^{2} a_{1}$ e $i \kappa\left|a_{2}\right|^{2} a_{2}$ são termos dissipativos não-lineares.

O modelo de ondas tipo sóliton (4) foi resolvido numericamente no domínio $\xi \times s=[0, T] \times$ $[-L, L]$, onde $T, L \in R$. Considerando as variáveis $a_{1}(\xi, s) \equiv a_{1}(k+1, j)$ e $a_{2}(\xi, s) \equiv a_{2}(k+1, j)$ para $k=0,1, \ldots, k_{\max }$ e $j=1,2, \ldots, n i$, onde $k_{\max }$ é denominado o último avanço em $\xi$ e $n i$ o número máximo de pontos em $s$, o domínio fica definido por uma malha computacional discreta de $k_{\max } \times n i$ pontos, conforme a figura .

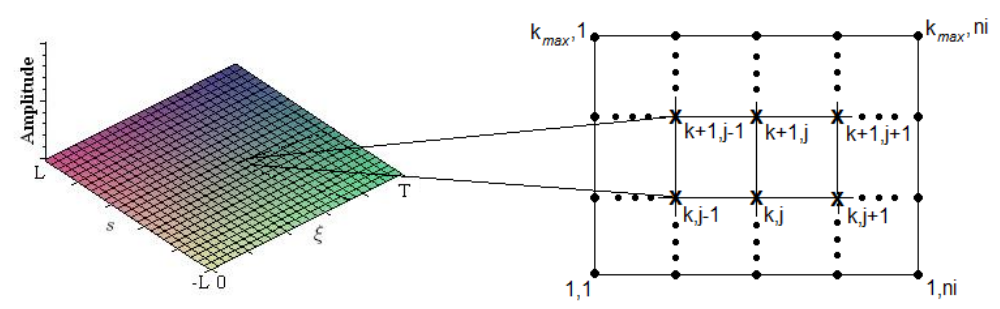

Figure 1: Domínio computacional para a propagação da onda sóliton.

O processo de discretização ocorrerá aproximando as derivadas temporais por diferenças finitas progressivas e as derivadas espaciais por diferenças finitas centrais e assim podemos reescrever (4) como:

$$
\begin{array}{r}
a_{1}(k+1, j)=\left(\frac{1}{{ }^{1} A_{p}}\right)\left[{ }^{1} A_{w} a_{1}(k+1, j-1)+{ }^{1} A_{e} a_{1}(k+1, j+1)+{ }^{1} A_{p_{0}} a_{1}(k, j)\right. \\
\left.-a_{1}^{*}(k, j) a_{2}(k, j) \exp (-i \beta \xi)+i \mu a_{1}(k, j)+i \kappa\left|a_{1}(k, j)\right|^{2} a_{1}(k, j)\right] \\
a_{2}(k+1, j)=\left(\frac{1}{{ }^{2} A_{p}}\right)\left[{ }^{2} A_{w} a_{2}(k+1, j-1)+{ }^{2} A_{e} a_{2}(k+1, j+1)+{ }^{2} A_{p_{0}} a_{2}(k, j)\right. \\
\left.-a_{1}^{2}(k+1, j) \exp (i \beta \xi)+i \mu a_{2}(k, j)+i \kappa\left|a_{2}(k, j)\right|^{2} a_{2}(k, j)\right]
\end{array}
$$

onde

$$
\begin{aligned}
{ }^{1} A_{p} & =i\left(\frac{1}{\Delta \xi}-\mu-\kappa\left|a_{1_{k+1, j}}\right|^{2}\right)+\frac{r}{(\Delta s)^{2}} & { }^{1} A_{e}={ }^{1} A_{w} \\
{ }^{1} A_{w} & =\frac{r}{2(\Delta s)^{2}} & { }^{1} A_{p_{0}}=\frac{i}{\Delta \xi} \\
{ }^{2} A_{p} & =i\left(\frac{1}{\Delta \xi}-\mu-\kappa\left|a_{2_{k+1, j}}\right|^{2}\right)+\frac{\alpha}{(\Delta s)^{2}} & { }^{2} A_{e}=\frac{\alpha}{2(\Delta s)^{2}}+\frac{i \delta}{2 \Delta s} \\
{ }^{2} A_{w} & =\frac{\alpha}{2(\Delta s)^{2}}-\frac{i \delta}{2 \Delta s} & { }^{2} A_{p_{0}}=\frac{i}{\Delta \xi} .
\end{aligned}
$$


Aplicando o Método de Gauss-Seidel calculamos iterativamente $a_{1}(k+1, j)^{n+1}$ por meio das equações:

$$
a_{1}(k+1, j)^{(n+1)}=\frac{{ }^{1} B_{j}+{ }^{1} A_{w} a_{1}(k+1, j-1)^{(n+1)}+{ }^{1} A_{e} a_{1}(k+1, j+1)^{(n)}}{{ }^{1} A_{p}}
$$

até que o critério de parada seja satisfeito:

$$
\max _{2 \leq j \leq n i-1}\left|a_{1}(k+1, j)^{(n+1)}-a_{1}(k+1, j)^{(n)}\right|<10^{-6},
$$

onde

$$
{ }^{1} B_{j}={ }^{1} A_{p_{0}} a_{1}(k, j)^{(n+1)}-a_{1}^{*}(k, j)^{(n+1)} a_{2}(k, j)^{(n+1)} \exp (-i \beta \xi) .
$$

Neste caso, somente soluções numéricas são possíveis, já que a onda propagada não é um sóliton. Analogamente calcula-se $a_{2}(k+1, j)^{(n+1)}$.

Em todas as simulações assumimos para os parâmetros dielétricos os seguintes valores: $r=$ $-1, \beta=-0,5, \alpha=-0,25$ e $\delta=-0,1$. Quanto à discretização, mantivemos $\Delta s=1,0 \times 10^{-1}$ e $\Delta \xi=1,0 \times 10^{-3}$ para as variáveis $s$ e $\xi$, respectivamente. Os intervalos de variação de $s$ e $\xi$ foram adaptados à cada caso de modo que não interferissem na plotagem dos gráficos. As figuras 2 e 3 a seguir mostram a propagação dos modos $a_{1}$ e $a_{2}$ do pacote de ondas sóliton em condições ideais, sem termos dissipativos, ou seja, com $\mu=\kappa=0$.
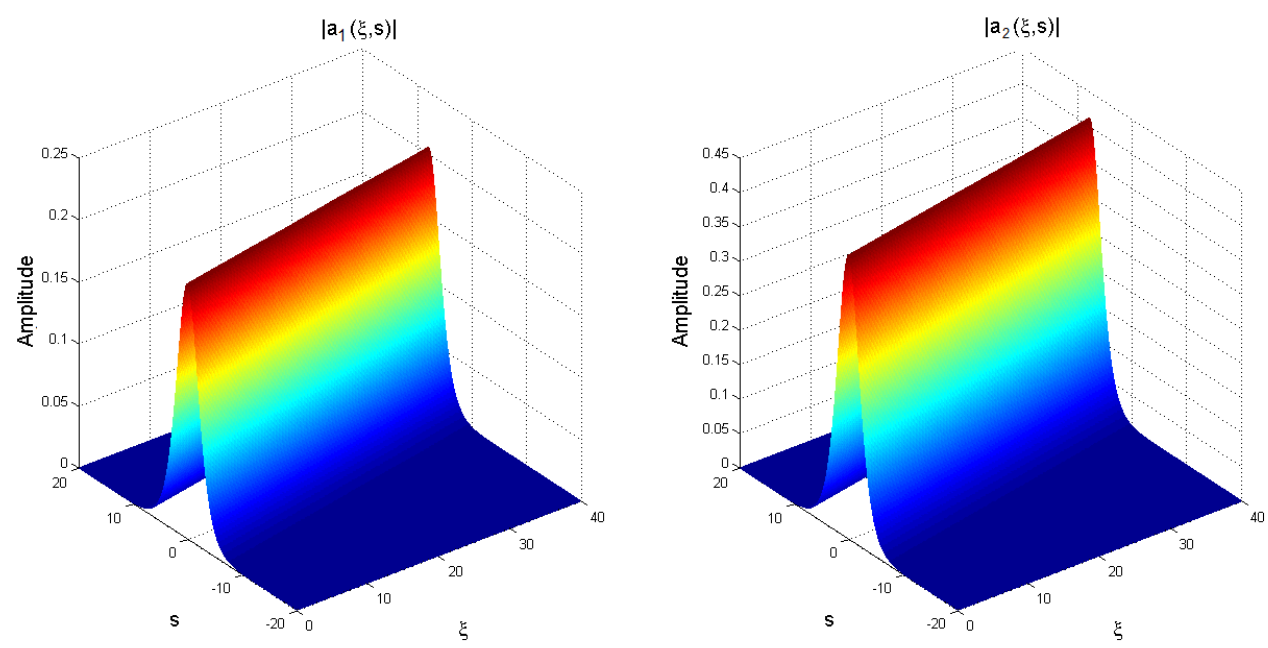

Figure 2: Propagação dos modos $a_{1}$ e $a_{2}$ da onda sóliton ideal.
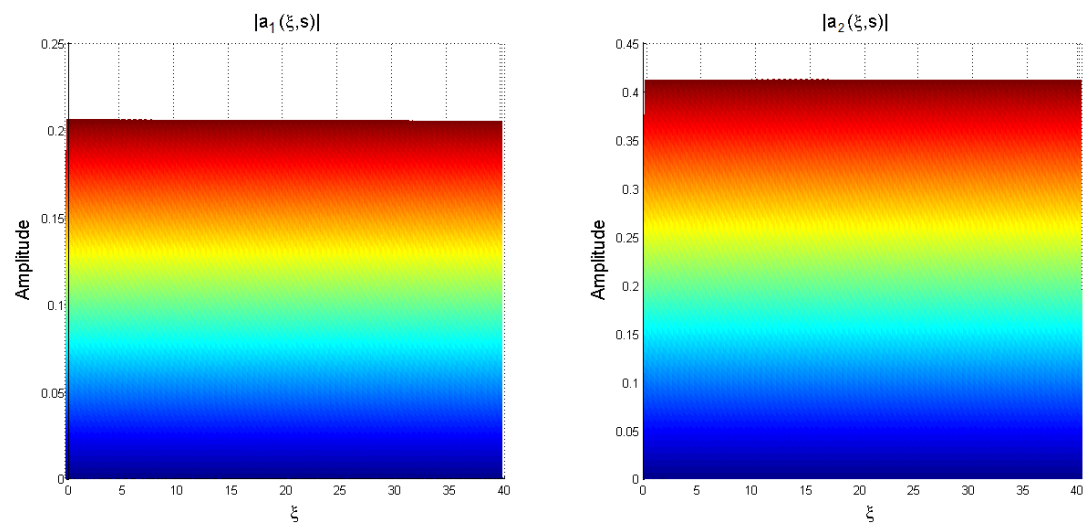

Figure 3: Perfil dos modos $a_{1}$ e $a_{2}$ da onda sóliton ideal. 
Note que a propagação ocorre sem que hajam distúrbios nas amplitudes das ondas, o que pode ser melhor observado na figura 3, onde visualiza-se a evolução das amplitudes em função da variável temporal $\xi$.

$\mathrm{Na}$ simulação seguinte acrescentamos o termo dissipativo linear $i \mu a_{n}$, com $n=1,2$, como apresentado no sistema de equações (4). Na figuras 4 e 5 tomamos $\mu=-1$ :
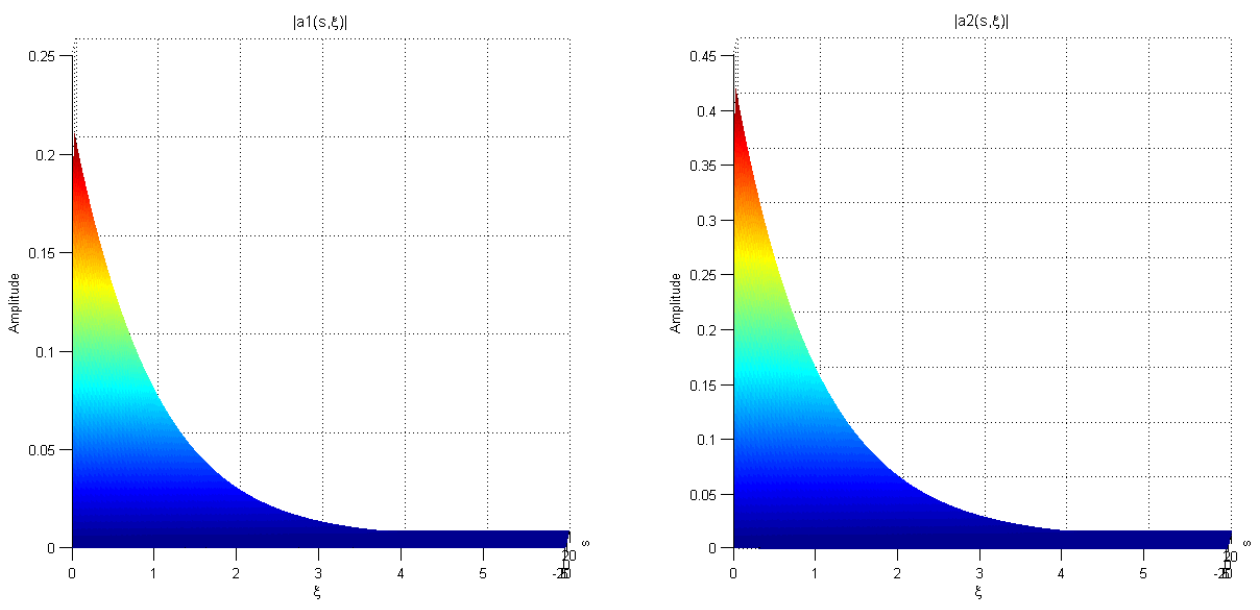

Figure 4: Perfil dos modos $a_{1}$ e $a_{2}$ da onda tipo sóliton com dissipação linear $\mu=-1$.

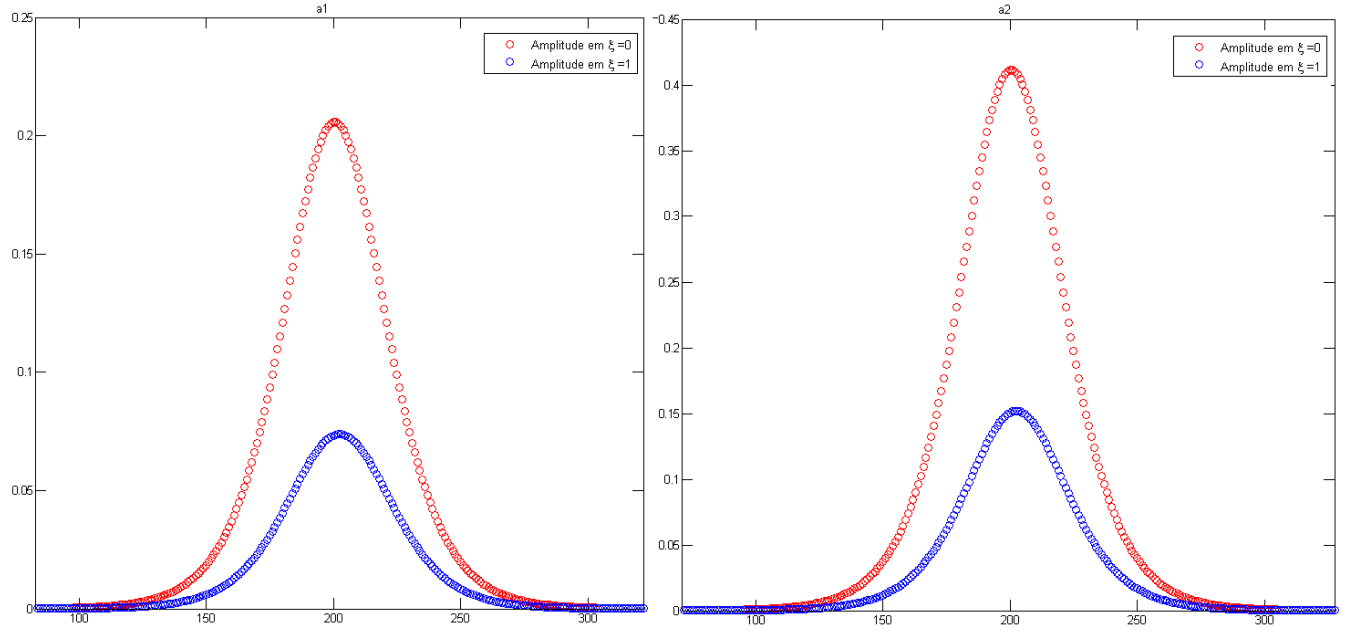

Figure 5: Cortes transversais de $a_{1}$ e $a_{2}$ para $\mu=-1$ em $\xi=0$ e $\xi=1$.

Note que o termo de dissipação linear atenua a onda sóliton, mas não gera dispersão (alargamento) dessa.

Nas próximas simulações incluímos o termo de dissipação não-linear $i \kappa\left|a_{n}\right|^{2} a_{n}$, com $n=1,2$. Nas figuras 6 e 7 fixamos o valor $\kappa=-1$. Verifica-se que taxa de decaimento do pacote de ondas nessas condições, com não-linearidades, é menos intenso que a taxa de decaimento devido à dissipação linear. Verifica-se também na figura 6 , que surgem oscilações nas amplitudes $a_{1}$ e $a_{2}$, provenientes da transferência de energia entre os modos acoplados em (1), devido ao parâmetro $\beta$. Finalmente, observa-se na figura 7, que o termo de dissipação não linear gera dispersão nas ondas. 

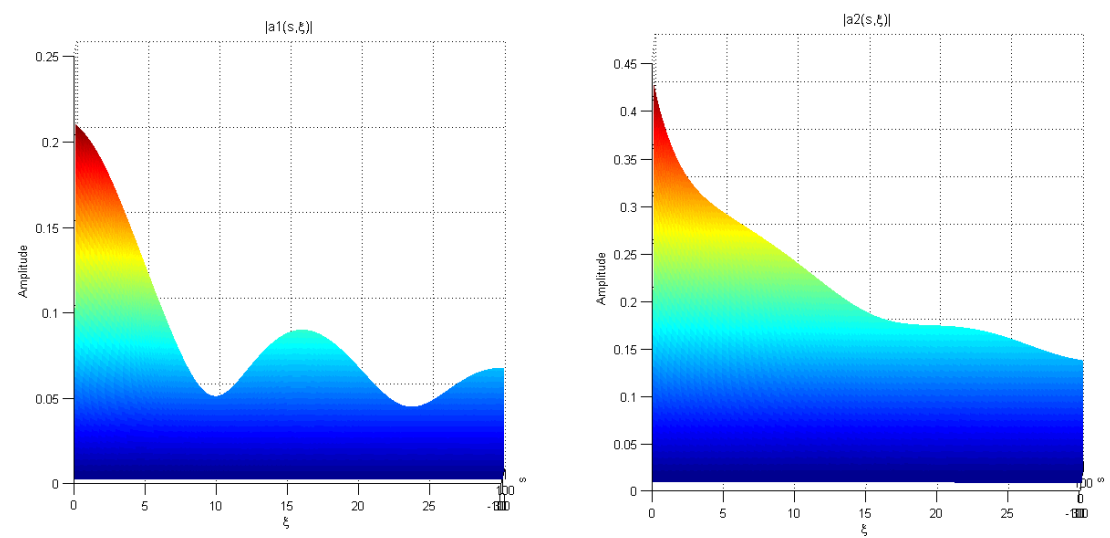

Figure 6: Perfil dos modos $a_{1}$ e $a_{2}$ da onda tipo sóliton com dissipação não linear $\kappa=-1$

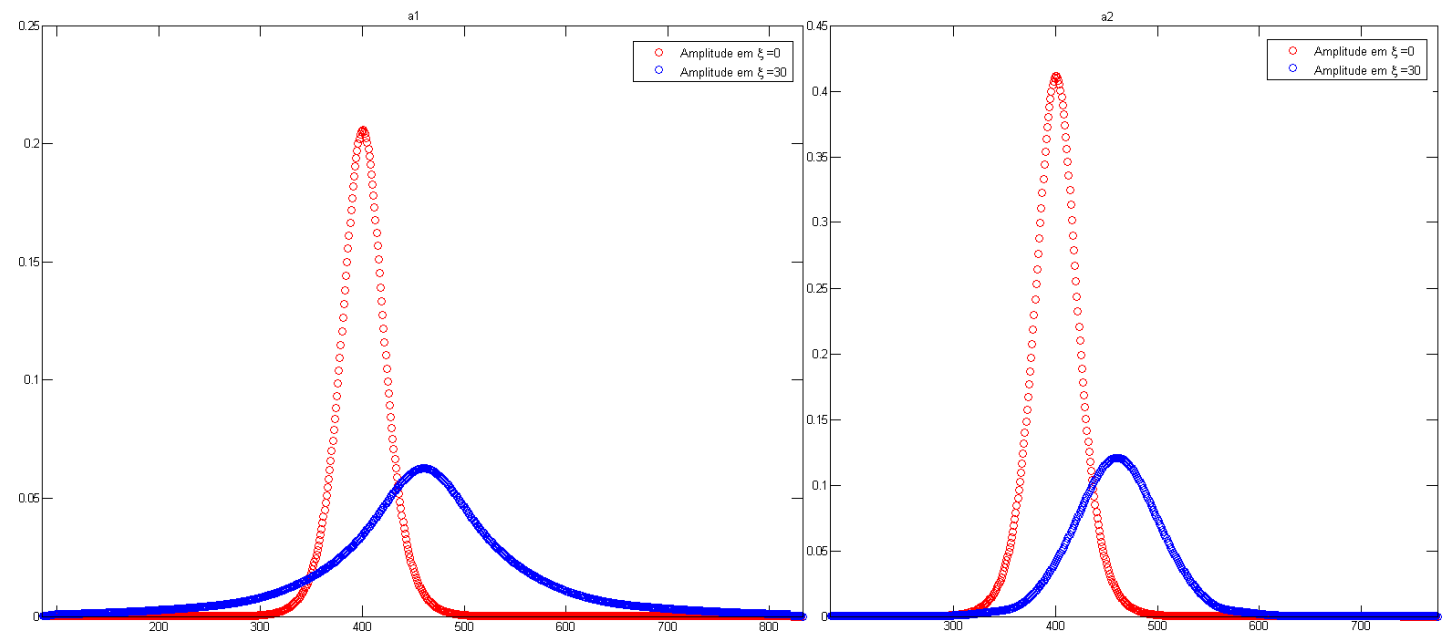

Figure 7: Cortes transversais de $a_{1}$ e $a_{2}$ para $\kappa=-1$ em $\xi=0$ e $\xi=30$.

\section{References}

[1] E. R. Cirilo, P. L. Natti, N. L. Romeiro, E. R. T. Natti, C. F. de Oliveira, Solitons in Ideal Optical Fibers - A Numerical Development, Semina Exatas, v. 31, p. 57-68, 2010.

[2] K. Fukuchi et al., 1.1-Tb/s (55 20-Gb/s) dense WDM soliton transmission over 3,020Km widely-dispersion-managed transmission line employing 1.55/1.58- $\mu \mathrm{m}$ hybrid repeaters, European Conference on Optical Communications (ECOC 99), PD2-10, France, 1999.

[3] W. Galléas, L. H. Ymai,P. L. Natti, E. R. T. Natti, Ondas do tipo sóliton em guias dielétricos, Revista Brasileira de Ensino de Física, v.25, p. 294-304, 2003.

[4] J. A. J. Ribeiro, Características da Propagação em Fibras Ópticas, Revista Inatel, v. 2, p. 1-16, 1999.

[5] N. J. Smith, F. M. Knox, N. J. Doran, K. J. Blow, I. Bennion, Enhanced power solitons in optical fibres with periodic dispersion management, Electronics Letters, v. 32, p. 54-55, 1996.

[6] L. H. Ymai, W. Galléas, P. L. Natti, E. R. T. Natti, Estabilidade de sólitons em fibras óticas dielétricas de tipo $\chi^{2}$, Revista Ciências Exatas e Naturais, v. 6, p. 9-29, 2004. 